\title{
Adipose tissue-derived mesenchymal stem cells ameliorate experimental acute colitis in rats
}

Seyed Jalil Masoumi

Shiraz University of Medical Sciences

Negar Hassanshahi

Shiraz University of Medical Sciences

Seyed-Mohammad Kazem Hosseini-Asl

Shiraz University of Medical Sciences

Davood Mehrabani ( $\nabla$ davood_mehrabani@yahoo.com )

Shiraz University of Medical Sciences https://orcid.org/0000-0002-5738-1719

Seyedeh-Sara Hashemi

Shiraz University of Medical Sciences

Amin Derakhshanfar

Shiraz University of Medical Sciences

Shahrokh Zare

Shiraz University of Medical Sciences

Iman Jamhiri

Shiraz University of Medical Sciences

\section{Research Article}

Keywords: adipose tissue, mesenchymal stem cells, ulcerative colitis, apoptosis

Posted Date: November 1st, 2021

DOI: https://doi.org/10.21203/rs.3.rs-1036129/v1

License: (c) (i) This work is licensed under a Creative Commons Attribution 4.0 International License.

Read Full License 


\section{Abstract}

Complete treatment of ulcerative colitis (UC) is still difficult, while conventional therapies have various adverse effects. Mesenchymal stem cells (MSCs) have anti-inflammatory and immunomodulatory properties to be a therapeutic candidate for UC. We evaluated therapeutic potential of adipose tissuederived mesenchymal stem cells (AdSCs) in treatment of an acute colitis rat model using histological and molecular assessments. Thirty male Sprague Dawley acetic acid-induced ( $2 \mathrm{~mL}$ of $3 \%$ ) acute colitis rat models were randomly divided into three equal groups of control receiving $0.5 \mathrm{~mL} / \mathrm{kg}$ of normal saline, sulfasalazine group receiving $500 \mathrm{mg} / \mathrm{kg}$ sulfasalazine and AdSCs group transplanted transrectally with $2 \times 10^{6} \mathrm{MSCs}$. They were evaluated histologically and by real time PCR for expression of apoptotic genes until 21 days. MSCs were spindle shape and positive for osteogenic and adipogenic differentiation. They displayed mesenchymal and lacked hematopoietic markers. In control group, severe inflammation, edema, ulcer, necrosis and infiltration of leukocytes were noticed. In sulfasalazine group, a moderate inflammation, edema, ulcer, necrosis and infiltration of leukocytes were visible; and in AdSCs group, mild inflammation, congestion, and infiltration of leukocytes were observed with a mild edema, but necrosis was absent in colonic tissue. A stronger decrease in expression of Bax, together with a higher increase in Bcl-2 was noted in AdSCs group. Based on histological and molecular findings, AdSCs were effective to ameliorate colitis lesions through their anti-inflammatory and anti-apoptotic activities showing that transplantation of AdSCS can be a potentially useful strategy in treatment of colitis.

\section{Introduction}

Inflammatory bowel diseases (IBDs) are a group of digestive diseases including ulcerative colitis (UC) and Crohn's disease (CD), while environmental, genetics, and immune factors have important roles in occurrence of the disease ${ }^{1}$. In 21 st century, IBD was reported a global burden of prevalent diseases, and hundreds of studies were conducted on incidence and prevalence of IBD throughout the world ${ }^{2}$. The prevalence of IBD has more stabilization in Western Europe and North America, but with a rise in Asia and developing countries ${ }^{3}$. Iran as one of the largest Asian countries in the Middle East which is adjacent to Turkey, Afghanistan, Pakistan, Central Asian and Persian Gulf countries, and they are a fertile ground for investigation for $\mathrm{IBD}^{3}$.

$\mathrm{UC}$ is a chronic and idiopathic inflammation in colon affecting many patients who suffer from a relapsing and remitting course ${ }^{4}$. Both male and female are equally influenced, specially enrolling 30-40 years old adults ${ }^{4}$. Inflammation of the lining of the colon happens in UC leading to several symptoms such as abdominal pain, diarrhea, and rectal bleeding ${ }^{4}$. The acute form of UC is a severe and life-threatening condition that conventional medications include anti-inflammatory agents such as systemic corticosteroids, topical corticosteroids, and 5-aminosalicylic acids (5-ASA), as well as immunomodulators like cyclosporine, azathioprine, 6-mercaptopurine (6-MP), and methotrexate ${ }^{5}$. It has endoscopic and surgical treatments too ${ }^{6}$, but unfortunately; complete treatment of UC is still difficult; because $74 \%$ of patients were reported to experience at least one relapse during a 5 -year period ${ }^{4}$. Taking conventional 
medications may lead to the occurrence of various adverse effects that can make nearly one fourth of patients discontinue their treatments ${ }^{4}$. Therefore, the global climbing trend of IBD would need a tandem effort to prevent the disease and to innovate the delivery of healthcare to patients with IBD and to introduce new therapeutic targets such as transplantation of mesenchymal stem cells (MSCs) to achieve ameliorative efficacy without a risk of incontinence ${ }^{4}$.

MSCs are multipotent stem cells that were widely investigated over the past few decades in several diseases ${ }^{7}$. They can be easily isolated and amplified from various tissues including bone marrow ${ }^{8}$ and adipose tissues ${ }^{9}$. MSCs can regulate immune responses, when exposed to inflammatory environment, thus promoting the repair and regeneration of damaged tissues ${ }^{10}$. They have been successfully used for treatment of UC in human and animals ${ }^{4}$. However, there are still very few studies regarding the efficiency of adipose tissue derived stem cells (AdSCs) as a candidate therapeutic strategy for UC. Therefore, the objective of this study was to determine the healing effect of AdSCs in experimental acute colitis of rat model that were evaluated histologically and quantitatively by real time PCR.

\section{Results}

\section{Cell Characterization}

AdSCs in different passages were adherent to the culture plates and were spindle shape (Fig. 1A, B). In osteogenic differentiation media, the cells illustrated calcium deposits after three weeks that were visualized in red color by Alizarin Red staining (Fig. 1C). In adipogenic differentiation media, the cells were stained by Oil Red-O and revealed intracellular lipid droplets in red color (Fig. 1D). Also, AdSCs displayed positive expression of mesenchymal markers (CD73 and CD90) and lacked expression of hematopoietic markers (CD34 and CD45) (Fig. 1E).

\section{Histological Assessment}

In control group 1 with colitis receiving normal saline treatment, inflammation, edema, conjestion, ulcer, necrosis and infiltration of leukocytes were severely visible in the colonic tissue (Fig. 2A) together with weight loss, hematochezia and bleeding, but without any mortality. In sulfasalazine group receiving sulfasalazine after induction of colitis, a moderate inflammation, edema, ulcer, necrosis and infiltration of leukocytes were confirmed with moderate weight loss, hematochezia and bleeding, but without any mortality (Fig. 2B and C). In cell transplantation group that AdSCs were injected for treatment of UC, mild inflammation, conjestion, and infiltration of leukocytes were observed with a mild edema, but necrosis, weight loss, hematochezia and bleeding were absent denoting to a prominent healing effect of stem cells in ameliorating UC (Fig. 2D-F). The strongest alterations were visible 3 weeks after cell transplantation.

\section{Quantitative Real Time PCR (qPCR)}


The significant decrease in expression of Bax pro-apoptotic gene was stronger in cell transplanted group when compared with the control and sulfasalazine-treated group. The highest changes were noticed 3 weeks after cell transplantation. The increase in Bcl-2 anti-apoptotic gene in AdSC transplanted group was significantly higher than other groups. The highest changes were noticed 3 weeks after cell transplantation (Fig. 3, $\mathrm{P} \otimes 0.05$ ).

\section{Discussion}

People who suffer from IBD are prone to increased risks of developing colorectal cancer, which has a global incidence rate and mortality rate ${ }^{4}$. During the last decade, conventional treatments for IBD were found to provide scarce desired findings and often with severe complications. Biological agents including transplantation of stem cells have been introduced as a treatment of choice in IBD with a great deal of success in both clinical and experimental models ${ }^{4}$. The ability of MSCs to actively proliferate, undergo plastic differentiation, exhibit low immunogenicity, trigger strong immune regulation, and express abundant trophic factors has confirmed their success in immune interventions and therapies and in regenerative medicine of $\mathrm{IBD}^{4}$.

In our study, the transplanted AdSCs were shown to have mesenchymal properties similar to previous reported findings ${ }^{9,10}$, that were plastic adherent, spindle shape, positive for osteogenic and adipogenic induction, and also positive for expression of CD73 and CD90 as mesenchymal markers and negative for expression CD34 and CD45 as hematopoietic markers. AdSCs can be easily isolated and expanded while maintaining their biological features ${ }^{9,10}$. Transplantation of MSCs in treatment of colitis has been reported before ${ }^{4,13}$. Currently, 7 undertaken case reports, 2 conducted clinical trials and 9 registered clinical trials are available in literature on application of MSCs in treatment of UC showing that MSCs have opened a new avenue in treatment of $\mathrm{UC}^{4,14}$. In case reports and clinical trials, successful use of bone marrow-derived stem cells (BMSCs) ${ }^{15}, \mathrm{AdSCs}^{16}$, peripheral blood mononuclear cells (PBMCs) ${ }^{17}$, umbilical cord-derived stem cells (UCSCs) ${ }^{18}$ and hematopoietic stem cells (HSCs) ${ }^{19}$ have been reported in alleviation of UC symptoms, and recovering from hematochezia and edema in the colon and rectum. Our results have also revealed that AdSCs were effective in reducing inflammation, congestion, edema, ulcer, necrosis and infiltration of leukocytes in the injured colonic tissue after induction of colitis. In our colitis model, AdSCs demonstrated anti-apoptotic effects too. The therapeutic and repairing impacts of MSCs after transplantation of cells in the damaged colonic tissue were shown by a decrease in expression of Bax and increase in Bcl-2 genes.

In addition to human studies, there are many in vivo studies on use of MSCs in treatment of UC in animal models. Various animals have been used as experimental model of UC and treatment of disease with MSCs including mouse in 39 studies $^{4,20}$, rat in 13 investigations ${ }^{4,21}$, Guinea pig in one study ${ }^{4,22}$ and dog in one study ${ }^{23}$. In our research, rat model has similarly been used for induction of colitis and AdSCs as a therapeutic approach with successful results of healing in colonic lesions. Among animal models, different methods were introduced for induction of UC with subsequent transplantation of MSCs in 
treatment of disease including acetic acid in one study ${ }^{4,24}$, dextran sulfate sodium (DSS) in 7 investigations ${ }^{4,25}$, and trinitrobenzene sulfonic acid (TNBS) in four assays ${ }^{4,26}$. Identically, we utilized acetic acid in our study to induce UC in the rat model resulting in colonic lesions of severe inflammation, edema, ulcer, necrosis and infiltration of leukocytes.

Among MSCs transplanted in animal models for treatment of UC, BMSCs in 24 studies [4, 25], AdSCs in 11 assessments ${ }^{4,26}$, UCSCs in 10 investigations ${ }^{4,27}$, amnion-derived stem cells (AMSCs) in 2 evaluations ${ }^{4,28}$, intestinal stem cells (ISCs) in 2 surveys ${ }^{4,29}$, placental mesenchymal stem cells $\left(\right.$ AFSCs) ${ }^{4,30}$, endometrial stem cells (EndSCs) ${ }^{4,31}$ and tonsil-derived stem cells (TSCs) each in one study have been utilized in treatment of colonic lesions ${ }^{4,32}$. In our study, AdSCs have been successfully used in treatment of acetic acid-induced colitis of rat model with amelioration of colonic lesions similar to previous reports ${ }^{4,26}$.

The therapeutic impacts of MSCs may be due to modulation in proliferation, differentiation and function of immunocytes; such as T cells, B cells, NK cells, dendritic cells (DCs), and macrophages ${ }^{33}$. MSCs maintaining a balance between M1 and M2 macrophages ${ }^{34}$, restore the lost balance between Treg cells and proinflammatory Th1/Th17 cells and recruit circulating leucocytes to trigger the macrophages and B cells in the injured colonic tissue. So the outcome would be a rise in cytokines such as IL-4, IL-10, IL-11, IL13 , and TGF- $\beta$, and a decline in cytokines such as IL-6, IL-12, IL-21, IL-23, and NF-KB activities ${ }^{35}$. It was shown that in the IBD microenvironment, an imbalance of T cell subsets happens ${ }^{18}$ and MSCs can attenuate colonic lesions by inducing apoptosis in T cells via the FAS ligand (FASL)-dependent pathway. They can also reduce the expression of 15-lox-1 in macrophages, enhance the expression of Foxp3, IL-10 mRNA and S1P pathway, suppress mucosal immune responses and inflammasome formation, downregulate pro-inflammatory cytokines, upregulate of FOXP3+ Treg cells, produce TSG-6 and secrete prostaglandin E2 to enhance amelioration of colonic lesions ${ }^{4,36}$.

In previous reports, the origin and type (autologous or allogeneic) of MSCs, method of administration (dosage, route, schedule, timing of infusions, the intervals between injections, pretreatment with chemokines or cytokines, etc.), animal modeling of colitis were illustrated to affect the treatment outcome 29,37 . The mode of storage can also be an influencing factor, while the storage in various laboratories regarding the cold chain, lyophilization, and transportation can be different. In our study, $2 \times 10^{6}$ autologous AdSCs were transplanted transrectally that could ameliorate colonic lesions, even there were some limitations in our study which are worthy of consideration including the assessment methods that were histological and molecular, the follow-up time that was for 21 days and the sample size that was 10 animals in each group.

In conclusion, based on histological and molecular findings, AdSCs were demonstrated to be effective in amelioration of colitis lesions through their anti-inflammatory and anti-apoptotic activities revealing that transplantation of AdSCS can be a potentially useful strategy in treatment of colitis. 


\section{Declarations}

Funding The authors would like to thank Shiraz University of Medical Sciences for financial support of this study which was master thesis project of Miss Negar Hassanshahi (IR,SUMS.REC.1398.056). We wish to appreciate kind collaboration of Stem Cell Technology Research Center and Comparative and Experimental Medicine Center of Shiraz University of Medical Sciences, Shiraz, Iran.

Conflict of interest The authors declare no conflict of interest for publication of this article.

\section{References}

1. Mehrabani, D. et al. Food avoidance in patients with ulcerative colitis: A review. Int J Nutr Sci 2, 189195, (2017).

2. $\mathrm{Ng}$, S.C. et al. Worldwide incidence and prevalence of inflammatory bowel disease in the $21 \mathrm{st}$ century: a systematic review of population-based studies. Lancet 390, 2769-2778, doi:10.1016/S0140-6736(17)32448-0 (2018).

3. Safarpour, A.R. et al. Epidemiology of inflammatory bowel diseases in Iran and Asia; A mini review. Iran J Med Sci 38, 140-149, (2013).

4. Hosseini-AsI S.K. et al. Therapeutic Effect of Mesenchymal Stem Cells in Ulcerative Colitis: A Review on Achievements and Challenges. J Clin Med 9, 3922, doi:10.3390/jcm9123922 (2020).

5. Dong, C. et al. Systematic review with meta-analysis: mortality in acute severe ulcerative colitis. Aliment Pharmacol Ther 51, 8-33, doi:10.1111/apt.15592 (2020).

6. Hassanshahi, N. et al. The effect of omega-3 fatty acids in ulcerative colitis: A systematic review. Int J Nutr Sci 3, 58-64, (2018).

7. Nazempour, M. et al. The effect of allogenic human Wharton's jelly stem cells seeded onto acellular dermal matrix in healing of rat burn wounds. J Cosmet Dermato/ 19, 995-1001, doi:10.1111/jocd.13109 (2020).

8. Iravani, K. et al. The healing effect of conditioned media and bone marrow-derived stem cells in laryngotracheal stenosis: a comparison in experimental dog model. World J Plast Surg 6, 190-197, (2017).

9. Mehrabani, D. et al. The growth kinetic, differentiation properties, karyotyping, and characterization of adipose tissue-derived stem cells in hamster. Comp Clin Pathol 25, 1017-1022, doi:10.1007/s00580-016-2300-7 (2016).

10. Mehrabani, D. et al. Comparison of the regenerative effect of adipose-derived stem cells, fibrin glue scaffold and autologus bone graft in experimental mandibular defect in rabbit. Dent Traumato/34, 413-420, doi:10.1111/edt.12435 (2018).

11. Khodakarm-Tafti, A. et al. Healing effects of elaeagnus angustifolia extract in experimentally induced ulcerative colitis in rats. J Pharmacol Toxico/ 10, 29-35, doi:10.3923/jpt.2015.29.35 (2015). 
12. Zare, S. et al. MRI-tTracking of dental pulp stem cells in vitro and in vivo using dextran-coated superparamagnetic iron oxide nanoparticles. J Clin Med 8, 1418, doi:10.3390/jcm8091418 (2019).

13. Ocansey, D.K. et al. The achievements and challenges of mesenchymal stem cell-based therapy in inflammatory bowel disease and its associated colorectal cancer. Stem Cell Int 2020, doi:10.1155/2020/7819824 7819824, (2020).

14. Nishikawa, T. et al. Filtrated adipose tissue-derived mesenchymal stem cell lysate ameliorates experimental acute colitis in mice. Dig Dis Sci 66, 1034-1044, doi:10.1007/s10620-020-06359-3 (2021).

15. Xiang, $\mathrm{H}$. et al. Autologous bone marrow stem cell transplantation for the treatment of ulcerative colitis complicated with herpes zoster: a case report. Front Med 10, 522-526, doi:10.1007/s11684016-0485-4 (2016).

16. Mizushima, T. et al. The features of adipose-derived stem cells in patients with inflammatory bowel diseases. Surg Today 48, 352-358, doi:10.1007/s00595-017-1591-x (2018).

17. Su, J. et al. Interleukin-25 enhances the capacity of mesenchymal stem cells to induce intestinal epithelial cell regeneration. Am J Transl Res 9, 5320-5331, (2017).

18. Hu, J. et al. Safety and therapeutic effect of mesenchymal stem cell infusion on moderate to severe ulcerative colitis. Exp Ther Med 12, 2983-2989, doi:10.3892/etm.2016.3724 (2016).

19. Kniazev O.V. et al. Immune response to biological therapy of inflammatory bowel diseases. Ter Arkh 85, 55-9, (2013).

20. Lopez-Santalla, M. et al. Cell therapy with mesenchymal stem cells induces an innate immune memory response that attenuates experimental colitis in the long term. J Crohns Colitis 14, 14241435, doi:10.1093/ecco-jcc/jjaa079 (2020).

21. Hassanshahi, N. et al. The healing effect of Aloe vera gel on acetic acid-induced ulcerative colitis in rat. Middle East J Dig Dis 12, 154-61, doi:10.34172/mejdd.2020.177 (2020).

22. Robinson, A.M. et al. The neuroprotective effects of human bone marrow mesenchymal stem cells are dose-dependent in TNBS colitis. Stem Cell Res Ther 8, 87, doi:10.1186/s13287-017-0540-3 (2017).

23. Mehrabani, D. et al. The comparison of two methods of steroid therapy via appendicostomy and enema in experimental acetic acid induced ulcerative colitis in dog. World App/ Sci J 26, 1538-1545, doi:10.5829/idosi.wasj.2013.26.12.8188 (2013).

24. Wang, M. et al. Intraperitoneal injection (IP), Intravenous injection (IV) or anal injection (AI)? Best way for mesenchymal stem cells transplantation for colitis. Sci Rep 6, 30696, doi:10.1038/srep30696 (2016).

25. Mashhouri, S. et al. Non-Adherent Bone Marrow-Derived Mesenchymal Stem Cells Ameliorate Clinical Manifestations and Inflammation in an Experimental Model of Ulcerative Colitis in Rats. Iran J Med Sci 45, 341-351, doi:10.30476/ijms.2020.72514.0 (2020).

26. Yousefi-Ahmadipour, A. et al. Combination therapy of mesenchymal stromal cells and sulfasalazine attenuates trinitrobenzene sulfonic acid induced colitis in the rat: The S1P pathway. J Cell Physiol 
234, 11078-11091, doi:10.1002/jcp.27944 (2019).

27. Chang, Y.L. et al. Therapeutic Efficacy of Subcutaneous and Intraperitoneal Injections of a Single Dose of Human Umbilical Mesenchymal Stem Cells in Acute and Chronic Colitis in a Mouse Model. $J$ Med Biol Eng 40, 82-90, doi:10.1007/s40846-019-00494-7 (2020).

28. Miyamoto, S. et al. Therapeutic effects of human amnion-derived mesenchymal stem cell transplantation and conditioned medium enema in rats with trinitrobenzene sulfonic acid-induced colitis. Am J Transl Res 9, 940-952, (2017).

29. Watanabe, S. et al. Conditioned mesenchymal stem cells produce pleiotropic gut trophic factors. $J$ Gastroentero/ 49, 270-82, doi:10.1007/s00535-013-0901-3 (2014).

30. Duan, L. et al. Extracellular vesicles derived from human placental mesenchymal stem cells alleviate experimental colitis in mice by inhibiting inflammation and oxidative stress. Int J Mol Med 46, 15511561, doi:10.3892/ijmm.2020.4679 (2020).

31. Yu, D. et al. Downregulation of Dickkopf-1 augments the therapeutic effects of endometrial regenerative cells on experimental colitis. Res Square 2020, 1-33, doi:10.21203/rs.3.rs-27576/v1 (2020).

32. Lee, K.E. et al. The efficacy of conditioned medium released by tonsil-derived mesenchymal stem cells in a chronic murine colitis model. PLoS One 14, e0225739, doi:10.1371/journal.pone.0225739 (2019).

33. Nikolic, A. et al. Intraperitoneal administration of mesenchymal stem cells ameliorates acute dextran sulfate sodium-induced colitis by suppressing dendritic cells. Biomed Pharmacother 100, 426-432, (2018)

34. Song, W.J. et al. TSG-6 released from intraperitoneally injected canine adipose tissue-derived mesenchymal stem cells ameliorate inflammatory bowel disease by inducing M2 macrophage switch in mice. Stem Cell Res Ther 9, 91, doi:10.1016/j.biopha.2018.02.060 (2018).

35. Neurath, M.F. Cytokines in inflammatory bowel disease. Nat Rev Immunol 14, 329-342, doi:10.1038/nri3661 (2014).

36. Kang, J. et al. hucMSCs Attenuate IBD through Releasing miR148b-5p to Inhibit the Expression of 15lox-1 in Macrophages. Mediators Inflamm 2019, 6953963, doi:10.1155/2019/6953963 (2019).

37. Grégoire, C. et al. Review article: mesenchymal stromal cell therapy for inflammatory bowel diseases. Aliment Pharmacol Ther 45, 205-221, doi:10.1111/apt.13864 (2017).

\section{Methods}

\section{Animals and Grouping}

In 2019, thirty male Sprague Dawley rats $(220 \pm 20 \mathrm{~g})$ that were purchased from Experimental and Comparative Medicine Center of Shiraz University of Medical Sciences, Shiraz, Iran were randomly divided into three equal groups of 10 animals. Control group received $0.5 \mathrm{~mL} / \mathrm{kg}$ of normal saline transrectally after induction of acute colitis. In sulfasalazine group, $500 \mathrm{mg} / \mathrm{kg}$ of sulfasalazine dissolved 
in $0.5 \mathrm{~mL}$ normal saline was administered transrectally after inducing acute colitis. In cell treatment group, $2 \times 10^{6}$ of rat AdSCs of passage $4^{\text {th }}$ in $0.5 \mathrm{~mL}$ volume were transplanted transrectally. Acute colitis was induced via enema using a needle inserted up to $8 \mathrm{~cm}$ into the colonic tissue and by injection of 2 $\mathrm{mL}$ of $3 \%$ acetic acid solution, while rats were kept in a vertical position (head-down) to avoid any leakage of acetic acid as described before ${ }^{11}$. To confirm induction of colitis, rats were checked after 24 hours to suffer from diarrhea or rectal bleeding. Prior to induction of colitis, rats were deprived from food intake for $24 \mathrm{~h}$, but had free access to water.

All treatment interventions were started 24 hours after induction of colitis. During interventions, rats were anaesthetized by intra-peritoneal injection of $3 \mathrm{mg} / \mathrm{kg}$ xylazine (2\% Rompun, Bayer Co., Germany) and 30 $\mathrm{mg} / \mathrm{kg}$ ketamine (Imalgène 1000, Merial, Germany). They were kept in separate cages under an ambient temperature of $21 \pm 2^{\circ} \mathrm{C}$ and a $65-70 \%$ relative humidity in a good ventilated room. All groups were followed for 21 days. On $7^{\text {th }}, 14^{\text {th }}$ and $21^{\text {st }}$ days following treatment measures, the animals were sacrificed, and tissue samples were provided from the distal $10 \mathrm{~cm}$ portion of the colon for histological and molecular assessments. The animal studies were undertaken based on NIH Guidelines for the Care and Use of Laboratory Animals. The experimental protocol was approved by the Institutional Animal Care and Use Committee of our University (1398.056). All methods conducted in this study regarding the animals were in accordance with the ethical standards of Institutional Animal Care and Use Committee of Shiraz University of Medical Sciences.

\section{Isolation of AdSCs}

Adipose tissues around the testes of rats were used for isolation of AdSCs. The isolated tissue was placed on ice under sterile condition and transferred to stem cell laboratory of Stem Cell Technology Research Center of Shiraz University of Medical Sciences, Shiraz, Iran. Briefly, adipose tissue was minced into tiny segments and was treated with $0.2 \%$ collagenase type II (Gibco, U.S.A.) for 40 min at $37^{\circ} \mathrm{C}$, while shaken. The lysed tissue was centrifuged at $1500 \mathrm{rpm}$ for $5 \mathrm{~min}$. The precipitate was suspended in $2 \mathrm{~mL}$ of Dulbecco's Modified Eagles Medium (DMEM; Gibco, USA) in culture flasks containing $3 \mathrm{~mL}$ DMEM supplemented with $10 \%$ fetal bovine serum (FBS, Gibco, USA), $1 \%$ penicillin and $1 \%$ streptomycin (Sigma, USA) and $2 \mathrm{mM} \mathrm{L-glutamine} \mathrm{(Invitrogen,} \mathrm{Netherlands)} \mathrm{and} \mathrm{were} \mathrm{put} \mathrm{in} \mathrm{an} \mathrm{incubator} \mathrm{containing} 5 \% \mathrm{CO}_{2}$ at $37^{\circ} \mathrm{C}$ and saturated humidity. After 3 days, the media was refreshed to reach $85 \%$ confluence. The cells were later sub-cultured until passage $4^{\text {th }}$ using $0.25 \%$ trypsin (Gibco, USA), while they were later inactivated by adding equal volume of DMEM.

The isolated AdSCs in passage $4^{\text {th }}$ were later cryopreserved to be used for future cell transplantation purposes using $2 \times 10^{6}$ viable cells $/ \mathrm{mL}$ in 50\% DMEM media, 40\% FBS, and $10 \%$ dimethyl sulfoxide (DMSO; MP Bio) in sterile labeled cryovials and kept in a nitrogen tank. To prepare for cell transplantation, they were taken out from nitrogen tank and transferred into a $37^{\circ} \mathrm{C}$ water bath for thawing. Centrifugation was carried out for $5 \mathrm{~min}$ at $1500 \mathrm{rpm}$ and the cell pellet was re-suspended in DMEM and placed in a $\mathrm{CO}_{2}$ incubator at $37^{\circ} \mathrm{C}$ and saturated humidity until use. 


\section{Characterization of AdSCs by Morphology}

AdSCs were assessed to be morphologically spindle shape.

\section{Characterization by Osteogenic Induction}

Approximately $5 \times 10^{4}$ AdSCs were transferred into 6-well plates, while the media was refreshed with osteogenic medium at $90 \%$ confluence containing the culture media supplemented with $15 \%$ FBS, 100 nM dexamethasone (Sigma-Aldrich, USA), $50 \mu \mathrm{M}$ ascorbic acid (Merck, Germany), and $10 \mathrm{mM}$ glycerol 3phosohate (Merck, Germany) for 21 days. The media change was done every 3 days and after 21 days, $10 \%$ formalin was added for 20 min to fix the cells. After 3 washes with deionized water, the differentiation was assessed by alizarin red staining (Sigma-Aldrich, USA) bound to calcium mineralized deposits and revealed a red color.

\section{Characterization by Adipogenic Induction}

Around $5 \times 10^{4}$ AdSCs were seeded in 6-well plates, while media change happened with adipogenic medium at $90 \%$ confluence using culture media supplemented with $15 \%$ FBS, $100 \mathrm{nM}$ dexamethasone, $100 \mu \mathrm{M}$ ascorbic acid, and $200 \mu \mathrm{M}$ indomethacin (Sigma-Aldrich, USA) for 21 days. After 3 weeks, 10\% formalin was added for $20 \mathrm{~min}$ to fix the cells and after 3 washes with deionized water, fresh $0.5 \%$ Oil Red-0 dye (Sigma-Aldrich, USA) dissolved in 2-propanol solution (Merck, Germany) for $2 \mathrm{~h}$ was added for staining of differentiated cells. Oil red $\mathrm{O}$ staining reveals red color droplets when adipogenic induction is positive.

\section{Characterization of AdSCs by Flow Cytometry}

CD73 and CD90 (Dako, Denmark) were used to confirm the positive expression of mesenchymal surface markers and CD34 and CD45 (Dako, Denmark) to verify the absence of hematopoietic surface markers.

\section{Histological Evaluation}

The removed colonic tissue provided from animals was transferred into $10 \%$ buffered formaldehyde for $72 \mathrm{~h}$. Then, dehydration was done by cold ethanol, and clearing was undertaken using cold xylene. The tissue samples were later embedded in paraffin at $53^{\circ} \mathrm{C}$ and a $5-\mu \mathrm{m}$ thickness tissue section was prepared serially, dried at $37^{\circ} \mathrm{C}$ for an hour and stained by hematoxylin and eosin (H\&E). All slides were visualized under a light microscope and photography was undertaken.

\section{RNA Extraction of AdSCs}

RNA extraction of AdSCs was done using an RNA extraction kit (Cinna Gen Inc., Tehran, Iran). The RNA was assessed at optical density ratio of A260/A280 and A260/A230 using a Nanodrop spectrophotometer (Nanodrop; Thermo Fisher Scientific,Waltham, USA). cDNA was prepared using 1000 ng total RNA applying the Revert Aid first strand cDNA synthesis kit (Thermo Fisher Scientific, Waltham, 
USA). Collection of the samples for analyzing the gene expression was based on methods described before $^{12}$.

\section{Quantitative Real Time Polymerase Chain Reaction (qPCR)}

The targeted apoptotic genes were $\mathrm{Bax}$ and $\mathrm{Bcl}-2$ and $\mathrm{B}_{2} \mathrm{~m}$ was considered as an endogenous control gene. The sequences of these genes were determined by NCBI database and primer sets were designed by primer3 software (Table 1). Real time PCR was carried out using SYBR Green I as reporter dye and Step One Real-Time PCR reactions (Applied Biosystems, Waltham, USA). In each reaction, $200 \mathrm{nM}$ of each primer was added to target the specific sequence. The PCR condition was set at $94^{\circ} \mathrm{C}$ for $10 \mathrm{~min}$ followed by 40 cycles at $94^{\circ} \mathrm{C}$ for $15 \mathrm{~s}$, at $60^{\circ} \mathrm{C}$ for $60 \mathrm{~s}$, and melting curve analysis ramping from 65 to $95^{\circ} \mathrm{C}$. The amplification signals of different samples were normalized to $\mathrm{B}_{2} \mathrm{~m}$ cycle threshold (Ct), and then the 2DDCt method was applied to compare mRNA levels of various groups, which represented a fold-change in data analysis ${ }^{12}$.

Table 1 The Bax, $\mathrm{Bcl}-2$ and $\mathrm{B} 2 \mathrm{~m}$ gene sequences designed by primer3 software

\begin{tabular}{|llrl|}
\hline Gene & Primer sequence & Size (bp) \\
\hline Bax & Forward: & 5'-CTGCAGAGGATGATTGCTGA-3' & 174 \\
& Reverse: & 5'-GATCAGCTCGGGCACTTTAG-3' & \\
\hline Bcl2 & Forward: & 5'-ATCGCTCTGTGGATGACTGAGTAC-3' & 134 \\
\hline & Reverse: & 5'-AGAGACAGCCAGGAGAAATCAAAC-3' \\
\hline B2m & Forward: & 5'-CGTGCTTGCCATTCAGAAA-3' & 244 \\
\hline & Reverse: & 5'-ATATACATCGGTCTCGGTGG-3' \\
\hline
\end{tabular}

bp: base pair.

\section{Statistical Analysis}

To compare the groups, one-way analysis of variance (ANOVA), Independent $t$ and Shapiro-Wilks tests and Prism software (GraphPad Software, version 6.0, San Diego, USA) were used. A $P$ value $<0.05$ was considered statistically significant.

\section{Figures}



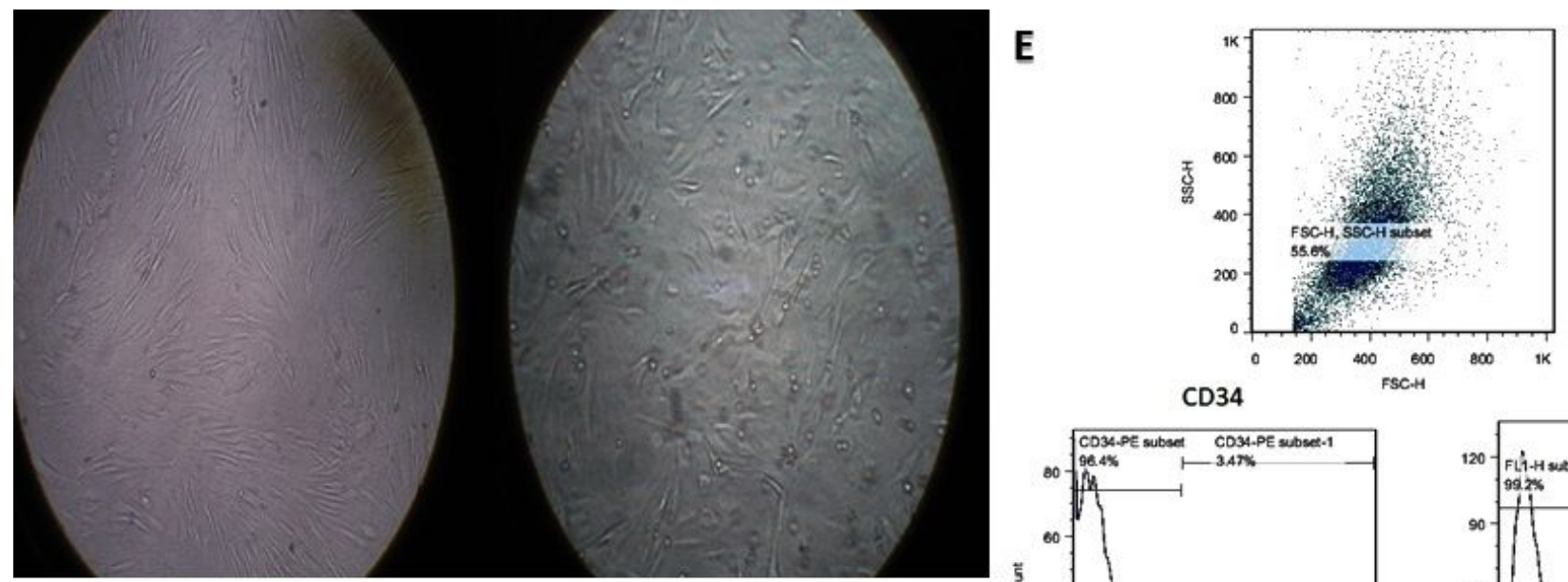

CD45
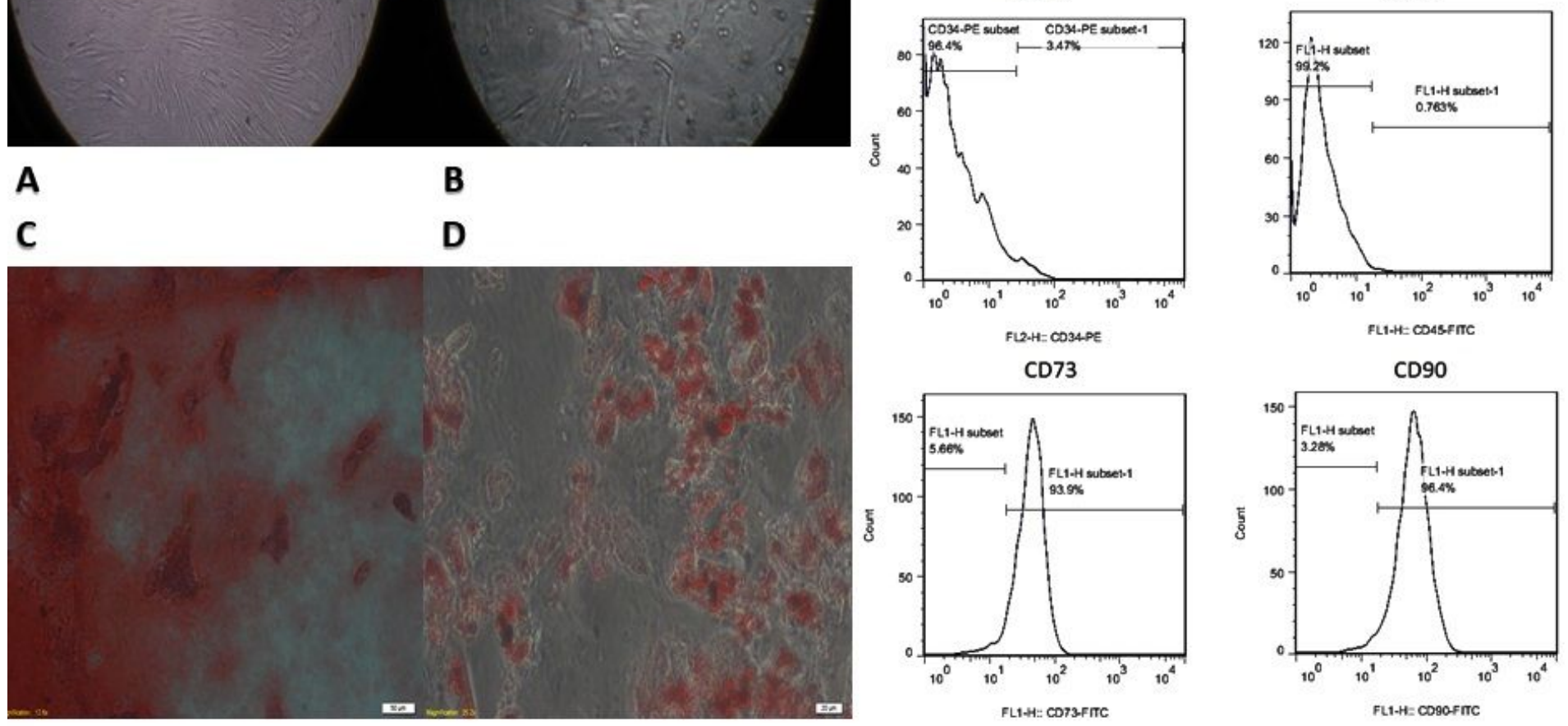

\section{Figure 1}

Characterization of AdSCs: A: 1st passage 1 (20x), B: 2nd passage (20x), C: Osteogenic induction in red color by Alizarin Red staining (20x), D: Adipogenic induction in red color by Oil Red 0 staining (20x), E: Flowcytometry being positive for expression of mesenchymal markers (CD73 and CD90) and negative for expression of hematopoietic markers (CD34 and CD45). 


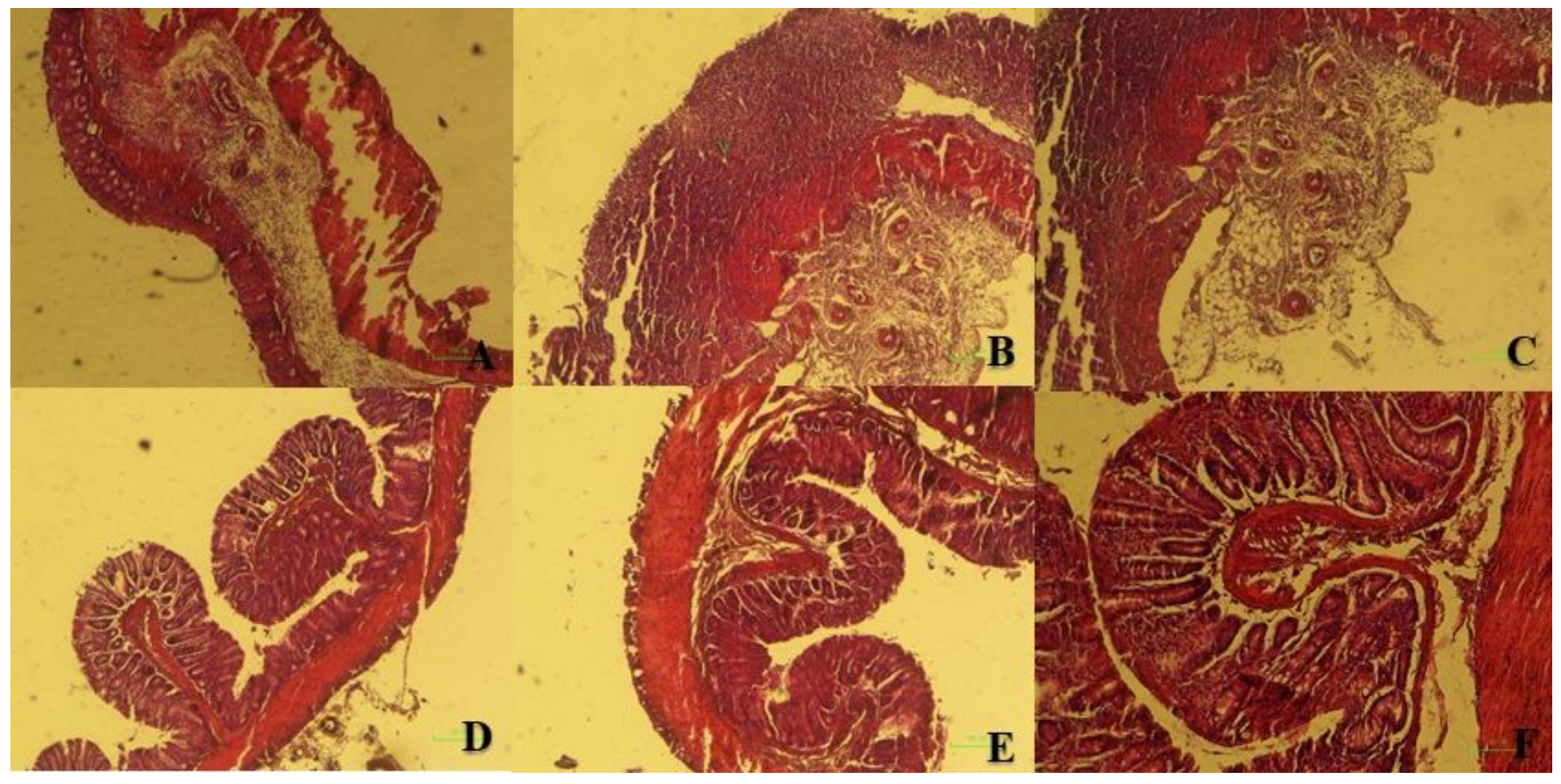

Figure 2

A. In control group receiving just normal saline for treatment, severe inflammation, edema, ulcer, necrosis and infiltration of leukocytes were noticed in the colonic tissue (H\&E, 40X). B and C. In sulfasalazinetreated group, a moderate inflammation, edema, ulcer, necrosis and infiltration of leukocytes were noted in the colonic injured tissue (H\&E, 40X). D-E. In AdSCs transplantation group, mild inflammation, congestion, and infiltration of leukocytes were observed with a mild edema, but necrosis was absent denoting to the prominent healing effect of stem cells in ameliorating UC.

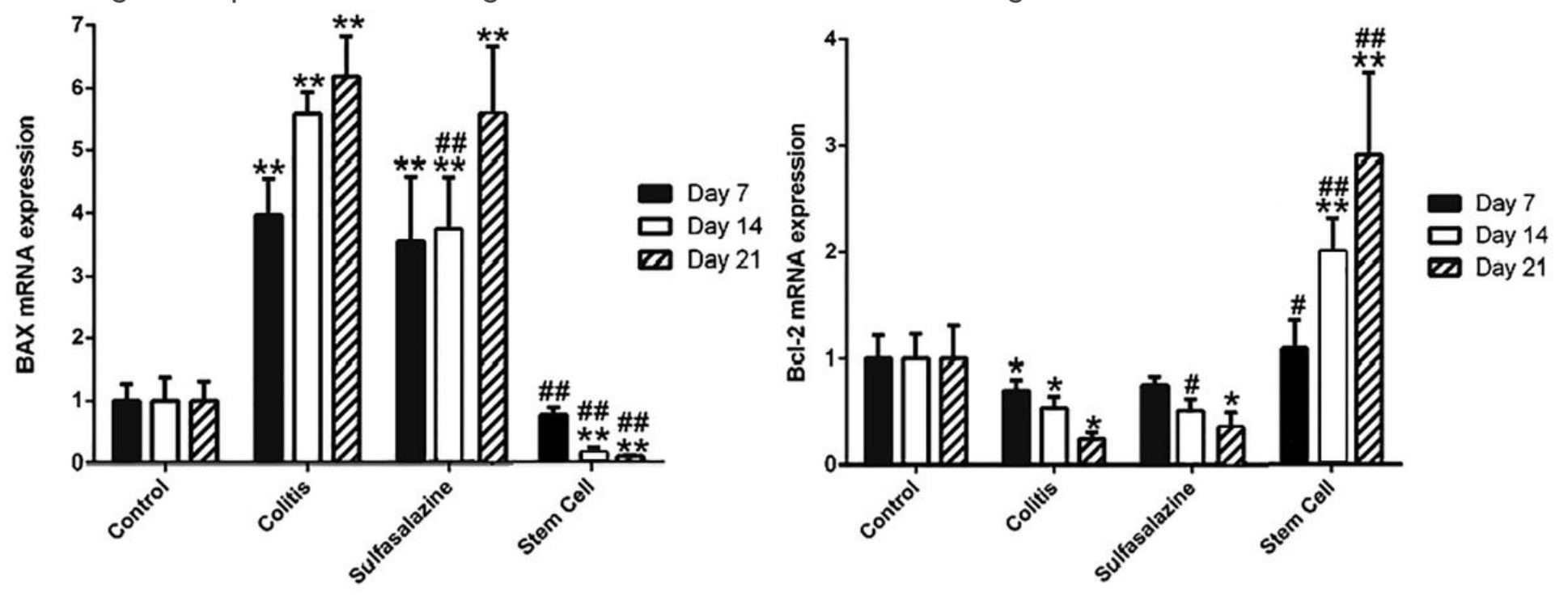

Figure 3 
The expression of Bax pro-apoptotic gene in AdSCs, and Bcl-2 anti-apoptotic gene compared between groups $\left({ }^{\star} \mathrm{P} \otimes 0.05\right)(\star \star P \otimes 0.001)(\# P \otimes 0.05)(\# \# P \otimes 0.001)$.

\section{Supplementary Files}

This is a list of supplementary files associated with this preprint. Click to download.

- Extendeddata20210931.docx 